\title{
DEVELOPMENT OF IOT ENABLED GLOBAL TRACKING SYSTEM AND MOBILE APPLICATION FOR PEOPLE WITH ALZHEIMER'S DISEASE
}

\author{
K. İleri ${ }^{1 *}$, A. Duru ${ }^{2}$, I.R. Karas ${ }^{3, a}$ \\ ${ }^{1}$ Dept. of Electrical and Electronics Engineering, Karabuk University, 78050 Karabuk, Turkey - kadirileri@ karabuk.edu.tr \\ ${ }^{2}$ Dept. of Biomedical Engineering, Karabuk University, 78050 Karabuk, Turkey - andayduru @karabuk.edu.tr \\ ${ }^{3}$ Dept. of Computer Engineering, Karabuk University, 78050 Karabuk, Turkey - ismail.karas@karabuk.edu.tr
}

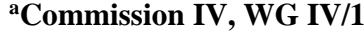

KEY WORDS: Internet of Things (IoT), GPS, Tracking System, Mobile Application, Wireless Network, Alzheimer's Disease.

\begin{abstract}
:
Alzheimer's is a degenerative disease meaning that it gets worse with time. Memory loss, speaking problems, wandering, and getting lost are some of the signs of the disease. The risk of wandering results in high demand for extensive monitoring solutions for the patients suffering from the disease. Tracking solutions are crucial, especially for family members and caregivers, so researchers develop new wearable tracking devices to overcome missing patients. GPS technology can provide location data with high accuracy, but it is not sufficient to use only by itself. Thus, a more extensive solution should be provided. In this paper, a mobile wearable tracking device that can provide data to the mobile application through internet has been developed for patient tracking purposes.
\end{abstract}

\section{INTRODUCTION}

Internet of Things (IoT) refers to internet-connected electronic objects that can collect and transfer data over a wireless network. In modern world, data can be transferred without any human intervention to another internet-connected device and they can share data with each other. This novel technology brings endless opportunities into the world and many applications benefits from it. Consumer, military, industrial, healthcare applications are some of the examples. Elder care, as an example, can benefit from assistance of smart homes. Smart homes can be equipped with assistive technologies and it can help elderly via voice control or smart device. Caregiver can also benefit from video camera in the home. Although there are ethical concerns about the video camera in the home, people living with dementia and their caregiver finds this technology useful (Mulvenna, M. et al. 2017). Falls or seizures can also be detected via camera effectively and dementia patients can live independently (Niemeijer, A. R., et al. 2011). People living with dementia and caregivers responses useful. Higher quality of life and freedom is possible with smart home technologies (Demiris, G., \& Hensel, B. K. 2008).

On the outside, one of the applications that can be used with IoT is a GPS tracking system (Mathur, Kalbande, 2020-Ramnath et al., 2017-Tang et al., 2016). The possibility of capturing realtime position data allows us to localize and detect moving objects with the use of GPS. GPS is a powerful tool to calculate the position on a global scale, especially for outdoor areas. Determination of position with GPS is very simple, the concept behind the positioning system is based on the measurement of time that takes for a signal to arrive from at least four satellites (Blewitt, 2007). On the other hand, GPRS technology allows mobility while connecting the device to the internet.

Although there are many GPS included devices in the market, portable, mini and wearable device is desired in our case because Alzheimer's patients has high risk of wandering (Neubauer et al., 2018). Patients with the disease can not recognize familiar places, so becoming lost, confusing about the location is common. Wandering may be dangerous and prevention is an important topic. Thus, Caregivers or relatives of the patient need to keep watch for any need, so a tracking system would be helpful. Remote overseeing not only for Alzheimer's patients but also can be used for elderly people or moving object tracking.

In this paper, we brought IoT and GPS Technologies together to design a mini tracking system for people with Alzheimer's disease. Wandering and getting lost are some of the signs of the disease, so the possibility of remote overseeing would help the caregivers and relatives of the patients. Therefore, the requirement of tracking patients keeps its importance to improve human life.

\section{SYSTEM DESIGN AND IMPLEMENTATION}

\subsection{Hardware Components}

The Microcontroller Unit (MCU): Arduino is an open-source electronics platform based on easy-to-use hardware and software. It is used for building electronics projects. There are several types of Arduino boards such as Uno, Nano, Micro, Due, and so on (Arduino, 2021). They are altered to adapt new requirements. Arduino is advantageous over other platforms because it is inexpensive, cross-platform capable, compatible with many sensors, easy to use software. They can be used for making variety of engineering projects using sensors such as temperature, obstacle, ultrasonic, GSM, pH, motion sensor, and so on. These sensor inputs can provide many flexible projects by turning it into outputs.

There are different platforms that can be used instead of Arduino such as Raspberry Pi, Odroid XU4, ASUS Tinker Board S, Nvidia Jetson Nano, and so on. They are capable of running multiple and more complicated programs instead of Arduino. However, Arduino is an easy-to-use board with lowpower consumption and lots of libraries to handle simple repetitive tasks. Hence, Arduino Uno is selected for this project.

GPS Module: Arduino Neo-6m GPS module which is shown in Figure 1 is chosen to acquire longitude and latitude data (Neo-

\footnotetext{
* corresponding author
} 
6m, u-blox, 2021). It is an inexpensive and low-power device, so this module is ideal for battery-operated mobile devices. The receiver works by calculating the distance from a number of satellites. It was pre-programmed about the positions of the satellites at any given time. Position of the satellites and current time information is sent by radio signals to the earth. These signals are received by the module and they make it possible to find its own position by calculating time differences. It has four pins which are positive power pin (VCC), ground (GND), UART receive pin (RX) and UART transmit pin (TX).

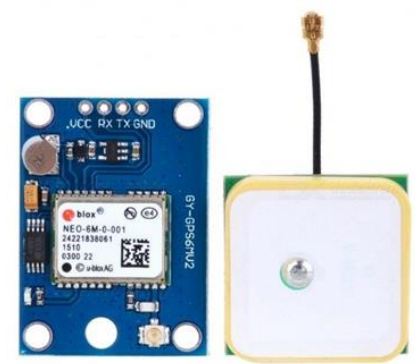

Figure 1. Neo-6m GPS module.

GSM/GPRS Module: SIM800L Mini GSM/GPRS module, shown in Figure 2, is a tiny cellular module that allows users to send/receive SMS, making/receiving calls and connect internet via Arduino by using GPRS transmission (Sim8001, Simcom, 2021). This module is a low cost, tiny product and perfect solution for any project that requires long-range connectivity thanks to quad-band frequency $(850 / 950 / 1800 / 1900 \mathrm{MHz})$ support. After powering up, it searches for the cellular network and $\log$ in automatically. It has twelve pins which are RING (LOW state while receiving a call), MICN (microphone, $\mathrm{N}$-), MICP (microphone, P +), SPKN (speaker, N -), SPKP (speaker, $\mathrm{P}+$ ), DTR (sleep mode, default in HIGH state), VCC (supply voltage), GND (ground), NET (antenna), RESET (reset), RXD (serial communication, receive pin) and TXD (serial communication, transmit pin).
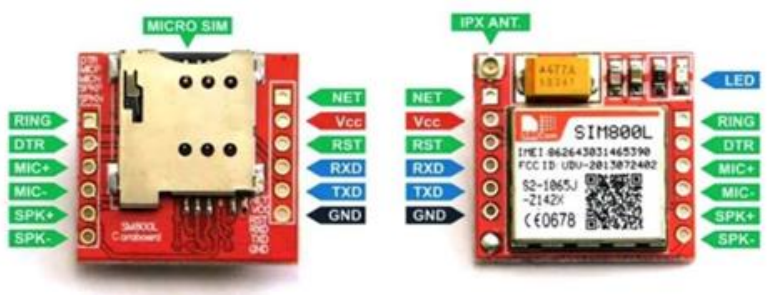

Figure 2. SIM800L Mini GSM/GPRS module.

\subsection{System Design}

The architecture of the designed system is shown in Figure 3. Chosen modules are connected considering data transfer and power pins. The system mainly consists of three parts which are portable setup, web server, and mobile application.

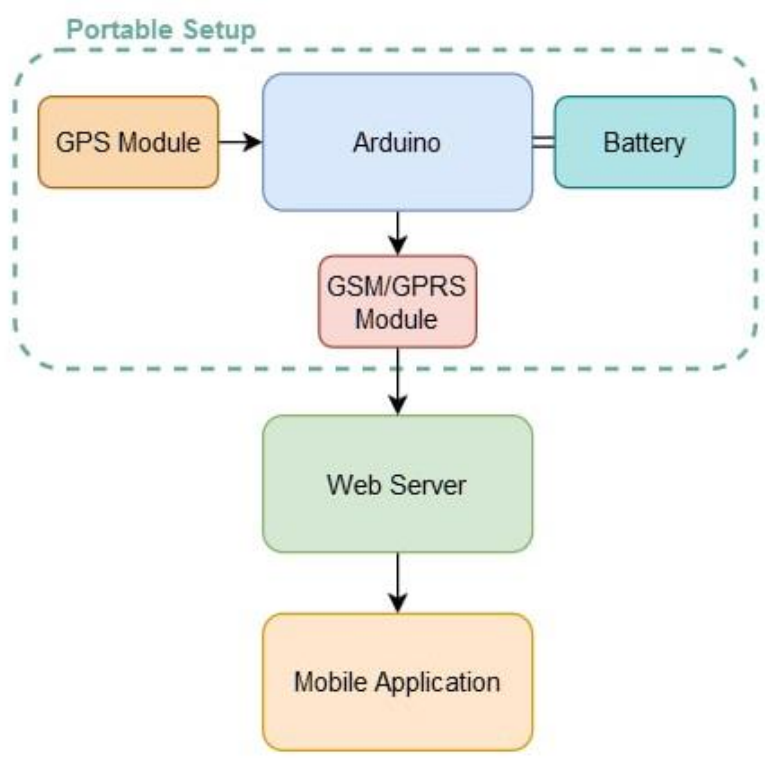

Figure 3. The architecture of the designed system.

The portable setup part is designed to find location of intended patients. It contains four parts which are Arduino, GPS module, GSM/GPRS module, and battery. Among those parts, GPS module provides the current location data by transferring latitude and longitude data to the microcontroller unit Arduino. However, the incoming data is in a raw form. Thus, the data has to be converted into readable form to display on the map. The data is converted on Arduino and sent to the GPRS module. GPRS module provides access and transfer data to the internet, so incoming data is sent to web server, periodically. The practical system of the portable setup is shown in Figure 4.

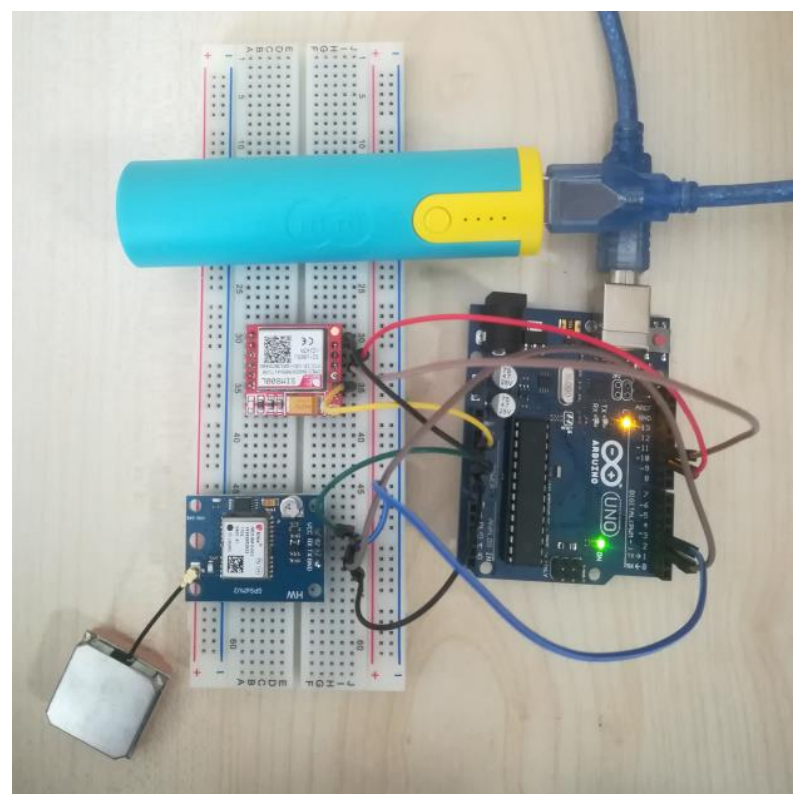

Figure 4. The practical system of the portable setup. 
The web server is the part that provides the communication between the portable setup and the mobile application. It gets the longitude and latitude data from the portable setup and uses these data to serve the mobile application.

The mobile application is the part that allows the caregiver or patient relative to track the patient remotely. It gets the longitude and latitude data from the webserver and shows the location of the patient on the map using these data.

\subsection{Development of the Mobile Application}

The mobile application that helps to track the patient remotely is developed by using Eclipse with Java programming language. Eclipse is an integrated development environment (IDE). It is mostly used for developing Java applications. However, it also supports to develop applications in other programming languages such as R, Python, Ruby, C, C++, PHP, Perl, Prolog, and so on with the help of plugins. Google Maps is embedded into the mobile application to show the patient location on the map. The location of the patient is marked on the map taking into account the data from the server.

The screenshots of the mobile application are shown in Figure 5. When the user starts the application, the screen in Figure 5a appears first. After the app opens, if the user presses the Show Location button, the location of the patient is shown on the map. Then, the location is tracked by the user as shown in Figure $5 \mathrm{~b}$.

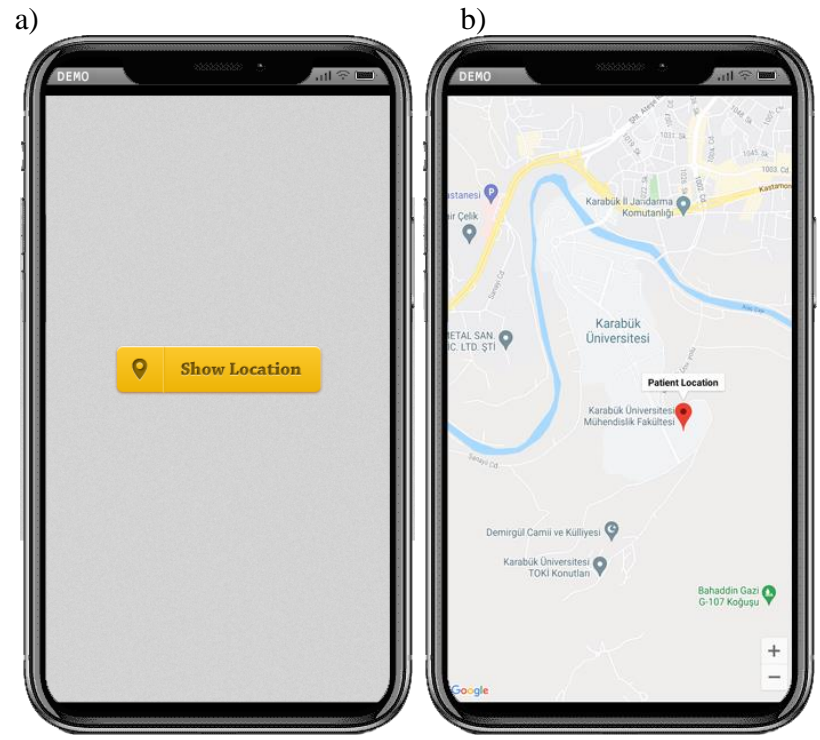

Figure 5. The screenshots of the developed mobile application.

The design of the developed mobile application is shown in Figure 6. The mobile application consists of two scenes which are Menu Scene and Map Scene. When the user starts the application, the Menu Scene first appears. The user can exit app via Exit button or open the Map Scene via Show Location button to check the location of the patient on the map. In Map Scene, the user can zoom in or out on the map to see the location clearly. Finally, the user can close the Map Scene and transition to Menu Scene via Back button to exit from the application.

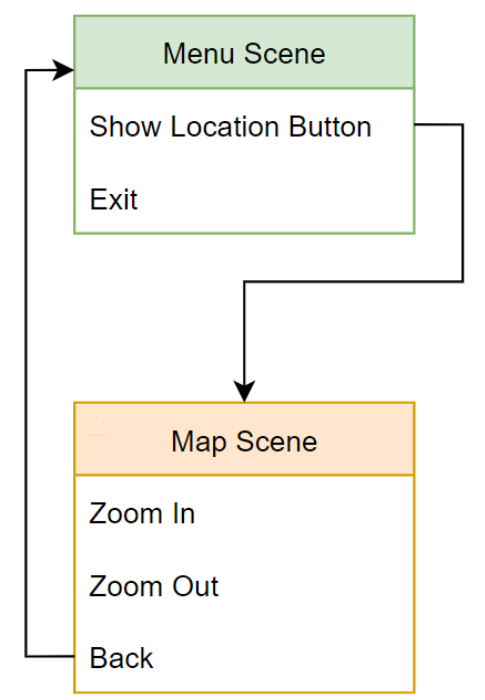

Figure 6. The design of the developed mobile application.

\section{CONCLUSION}

The proposed and implemented system design for finding and tracking people with Alzheimer's disease offers a new solution for wandering and getting lost cases. The system has been designed with existent technologies to develop an internetconnected mini tracking device. Internet connection of the tracking device provides instant access to location data. Thus, caregivers or relatives of the patients can get easy access to a map with the patient's coordinates from the developed mobile application. Moreover, it is important to point out that the only requirement is carrying the device for patients, there is no need for human interaction to send location data to the server. Since the device is small, it would be easier to attach it to the clothes or shoes. Future developments may include wake-up commands to increase battery life and making an enclosed single board with arduino nano for easier attachment to the clothes or shoes. Comfortable wearing is an important subject for securely carrying the device. Speaker may be adjusted for two way communication and it may support the patient. Smart alerts can be adjusted for being out of determined zone or help. By this way, quality of life will increase for both the patients and caregivers. On the other hand, the device can be used for other purposes. Vehicles can be tracked with this system and there will not be any battery problems because it can be directly powered up by battery of car. Luggages, bikes, valuables can also be tracked with this system.

\section{REFERENCES}

Demiris, G., \& Hensel, B. K. (2008). Technologies for an aging society: a systematic review of "smart home" applications. Yearbook of medical informatics, 17(01), 33-40.

Mulvenna, M., Hutton, A., Coates, V., Martin, S., Todd, S., Bond, R., \& Moorhead, A. (2017). Views of caregivers on the ethics of assistive technology used for home surveillance of people living with dementia. Neuroethics, 10(2), 255-266.

Niemeijer, A. R., Frederiks, B. J., Depla, M. F., Legemaate, J., Eefsting, J. A., \& Hertogh, C. M. (2011). The ideal application 
of surveillance technology in residential care for people with dementia. Journal of medical ethics, 37(5), 303-310.

Mathur, R., \& Kalbande, K. (2020). Internet of Things (IoT) based Energy Tracking and Bill Estimation System. 2020 Fourth International Conference on I-SMAC (Iot in Social, Mobile, Analytics and Cloud) (I-SMAC). doi: 10.1109/ismac49090.2020.9243480

Ramnath, S., Javali, A., Narang, B., Mishra, P., \& Routray, S. (2017). IoT based localization and tracking. 2017 International Conference on Iot and Application (ICIOT). doi: 10.1109/iciota.2017.8073629

Tang, H., Shi, J., \& Lei, K. (2016). A smart low-consumption IoT framework for location tracking and its real application. 2016 6Th International Conference on Electronics Information and Emergency Communication (ICEIEC). doi: 10.1109/iceiec. 2016.7589744

Blewitt, G. (2007). GPS and Space-Based Geodetic Methods. Treatise on Geophysics, 351-390. doi: 10.1016/b978044452748-6.00058-4

Neubauer, N., Azad-Khaneghah, P., Miguel-Cruz, A., \& Liu, L. (2018). What do we know about strategies to manage dementia-related wandering? A scoping review. Alzheimer's \& Dementia: Diagnosis, Assessment \& Disease Monitoring, 10(1), 615-628. doi: 10.1016/j.dadm.2018.08.001

Arduino - Home. (2021). Retrieved 25 August 2021, from https://www.arduino.cc/

NEO-6M, u-blox 6 GPS Modules, Data Sheet, Retrieved 25 August 2021, from https://www.u-blox.com/

SIM 800L, Small size quad-band GSM/GPRS module Data Sheet, Retrieved 25 August 2021, from https://www.simcom.com/ 